\title{
TV/Series
}

Hors séries 1 | 2016

Lost: (re)garder l'île

\section{Penser l'altérité avec Lost : Foucault, Lacan et la médiation du regard}

\section{Louis-Paul Willis}

\section{(2) OpenEdition \\ Journals}

Édition électronique

URL : http://journals.openedition.org/tvseries/1741

DOI : 10.4000/tvseries. 1741

ISSN : 2266-0909

Éditeur

GRIC - Groupe de recherche Identités et Cultures

Référence électronique

Louis-Paul Willis, «Penser l'altérité avec Lost: Foucault, Lacan et la médiation du regard », TV/Series [En ligne], Hors séries 1 | 2016, mis en ligne le 15 octobre 2016, consulté le 03 mai 2019. URL : http:// journals.openedition.org/tvseries/1741 ; DOI : 10.4000/tvseries.1741

Ce document a été généré automatiquement le 3 mai 2019.

\section{(c) (i) (9)}

TV/Series est mis à disposition selon les termes de la licence Creative Commons Attribution - Pas d'Utilisation Commerciale - Pas de Modification 4.0 International. 


\title{
Penser l'altérité avec Lost : Foucault, Lacan et la médiation du regard
}

\author{
Louis-Paul Willis
}

1 Il va sans dire que la série Lost laisse dans son sillage des répercussions considérables. À l'instar de certaines autres séries télévisuelles contemporaines, Lost a notamment eu un effet marquant sur les possibilités qu'offre la forme même du médium, à la fois sur les plans narratif, esthétique et thématique. Au niveau de la forme narrative, Lost semble même témoigner d'une tendance plus généralisée vers les narrations que nous pourrions qualifier de complexes ${ }^{1}$; la série met effectivement de l'avant une trame narrative dans laquelle le spectateur se doit de s'investir au plan cognitif s'il veut pouvoir reconstruire le sens des événements, ces derniers s'inscrivant simultanément dans un présent diégétique, un passé, un futur, et un présent parallèle. La série a également mené vers certaines innovations sur le plan méta-narratif, incluant entre autres des contenus en ligne, ainsi qu'un univers vidéoludique, pour déployer un univers diégétique à la fois complexe et captivant. Ces lieux d'innovation, défrichés par les créateurs de la série, suscitent de l'intérêt depuis la diffusion de la série en 2004, et beaucoup reste à faire afin de bien saisir la portée qu'aura eue cette fiction sur les publics tout comme sur l'histoire télévisuelle.

2 La portée qu'exerce la série sur son spectateur se déploie sur plusieurs fronts. Lost multiplie notamment les références philosophiques qui viennent enrichir la réception des téléspectateurs avertis. Par exemple, le nom de certains personnages est souvent évocateur de conflits existentiels entre différents systèmes de croyances. Mais si le lien entre Lost et la philosophie a amplement été soulevé jusqu'à présent, il reste que peu a été dit sur la série en termes psychanalytiques. Plus spécifiquement, force est de constater que la question de la vision, du regard et de leurs rôles dans la réception de la série demeure inexplorée et ce, malgré leur présence prépondérante dans la structure narrative de la série. Certes, on a noté les références à la philosophie de Michel Foucault, et particulièrement à la surveillance et au regard panoptique ${ }^{2}$. Sans doute serait-il tentant de limiter l'exploration de la question du regard à cette dimension foucaldienne. Or, une telle perspective négligerait certaines facettes plus radicales des réflexions sur le regard 
offertes par la psychanalyse. Parce que l'une des thématiques centrales de Lost demeure l'altérité, qui s'y décline sous plusieurs formes, il semble pertinent de se tourner vers la pensée du psychanalyste Jacques Lacan, d'autant plus que la relation entre le sujet et l'Autre y joue un rôle central.

Dans cette perspective, il s'agira ici d'examiner les répercussions spectatorielles liées à l'articulation du regard dans les six saisons de la série, afin de mieux comprendre la façon dont la dynamique du regard détermine certains des aspects les plus radicaux de Lost et, surtout, de sa réception par les spectateurs. Il sera d'abord question de mettre en rapport le regard panoptique foucaldien avec le regard dans sa conception psychanalytique, ellemême détaillée dans l'œuvre de Lacan. Par la suite, en tissant des liens entre ces deux regards déployés au champ de l'Autre, il sera possible d'aborder Lost comme un discours télévisuel qui explore la nature même de l'existence subjective à l'ère du panoptisme. En examinant la série à travers sa mise en scène de ces concepts, il sera possible de constater la radicalité qu'elle articule autour de la notion de l'altérité, radicalité qui passe par l'expression du regard médiatique auquel la série confronte ses spectateurs. La présente analyse se donne conséquemment comme objectif ultime de penser la série autour de sa réception par les spectateurs, eux-mêmes confrontés à une existence subjective marquée par l'altérité.

\section{Regard, vision}

4 Avant d'aborder la médiation du regard dans la série, un détour s'impose afin de mettre en avant une conceptualisation du regard dans son sens psychanalytique. La présente section vise donc à familiariser les lecteurs avec les enjeux théoriques entourant le regard et la déclinaison des dynamiques visuelles dans les médias contemporains, puisque certains de ces enjeux ont été jusqu'à présent absents des analyses proposées sur Lost ${ }^{3}$. La psychanalyse pense le regard d'une façon fort différente de la conception qui s'en dégage dans notre langage plus courant. En effet, le regard est à distinguer de la vision; plus spécifiquement, dans son sens psychanalytique, le regard se définit comme étant précisément l'opposé, ou l'antithèse, de la vision. Freud en vient à discerner la scopophilie ${ }^{4}$ active de la scopophilie passive; le sujet peut, en effet, prendre plaisir dans l'acte de voir et dans l'acte d'être $v^{5}$. Les études cinématographiques et médiatiques se sont longtemps concentrées uniquement sur l'idée d'une scopophilie active. Cette situation remonte aux premières réflexions sur le plaisir visuel, initiées par la féministe Laura Mulvey en 1975. Les spectateurs étaient alors imaginés comme des voyeurs prenant plaisir à s'immiscer dans une scène privée en tant qu'observateurs omnipotents détenant une maîtrise visuelle sur l'objet de la vision ${ }^{6}$. Avec ses plans fixes et ses cadrages calculés, le cinéma classique hollywoodien confère effectivement un réalisme aux scènes observées, tout en donnant dans le même temps aux spectateurs une vision irréaliste puisqu'elle ne renvoie pas leur propre vision subjective. Par le biais d'un usage purement réaliste du dispositif cinématographique, le cinéma populaire tente de plonger le spectateur dans une position qui se veut «toute-percevante ${ }^{7}$ », position bien entendue impossible. En effet, considérer le regard du spectateur comme un simple regard voyeur omet nécessairement la dimension beaucoup plus radicale de ce qui échappe à la vision : tout champ visuel contient un point mort, l'idée de quelque chose qui se donne à voir mais qui demeure inaccessible. Alors que la majorité des productions cinématographiques et télévisuelles populaires évitent de révéler ce point mort dans le champ du visible, 
certains discours médiatiques visuels cherchent à en révéler la présence afin de confronter le spectateur à une expérience de spectature plus radicale. Comme nous serons à même de le constater ultérieurement, Lost joue précisément sur cette dynamique visuelle plus inhabituelle.

5 En se fondant sur la conception du regard que propose Lacan, ainsi que sur son opposition à la simple vision, la radicalité potentielle du médium audiovisuel, qui est mise en avant dans Lost, devient beaucoup plus grande. En effet, Lacan situe le regard non pas dans l'acte de voir et de regarder, mais plutôt dans l'acte d'être vu et regardé. Faisant appel à la scopophilie passive de Freud, Lacan suggère que le regard déclenche le désir propre au champ scopique puisque «ce regard que je rencontre [...] est, non point un regard vu, mais un regard par moi-même imaginé au champ de l'Autre $»^{8}$. La théoricienne féministe Elizabeth Cowie opère d'ailleurs une distinction toute lacanienne entre regard et vision, spécifiant que «l'acte de voir relève de la simple vision, alors que le regard découle de la posture du sujet dans un champ de vision $»^{9}$. Cette distinction l'amène à affirmer que «le plaisir lié à la pulsion scopique est d'abord et avant tout passif - le désir d'être vu (dans son propre sexe, finalement) $»^{10}$. Le désir scopique - et par conséquent le désir qui motive les spectateurs de cinéma ou de télévision - est ainsi articulé non pas autour du contrôle que pourrait leur procurer le point de vue omniscient et panoptique, mais plutôt autour d'une impossibilité. Pour reprendre Cowie à nouveau, «Le désir ici n'est pas celui de voir quelque chose de particulier, ni même de voir, mais bien celui de voir ce qui est montré, ce que je ne peux encore voir mais que je sais être là à voir ${ }^{11}$ ». Le regard renvoie le sujet à la dimension radicale, insaisissable, de tout champ de vision; il n'est jamais possible de tout voir, et cette impossibilité mène à l'impression d'une perspective qui échappe, qui s'élide, et qui fait dérailler le sentiment de maitrise du sujet observant - un sentiment qui émerge régulièrement dans la série Lost, comme nous serons amenés à le découvrir. Comme le propose Todd McGowan, le regard lacanien «n'est pas la vision posée par le sujet sur l'objet, mais plutôt le point à partir duquel l'objet observé retourne le regard ${ }^{12}$ ». En somme, pour Lacan, le regard est un point mort dans le champ du visible, et ce point mort renvoie au sujet sa propre position comme objet dans une perspective qui lui est externe. Cette conception du regard se pose ainsi comme l'antithèse d'une vision purement omnisciente, et change l'idée qu'on peut se faire de la réception filmique et télévisuelle.

6 Il devient crucial à ce stade de mettre cette conception lacanienne du regard dans un rapport de tension avec l'idée que se fait Foucault du regard panoptique, et ce pour plusieurs raisons : d'emblée, un nombre considérable d'analyses de Lost se fondent sur l'apparente similitude entre l'expérience des survivants sur l'île et l'expérience panoptique; de plus, si l'importance de Foucault dans l'étude de Lost n'a plus besoin d'être démontrée, il en va tout autrement de l'importance pouvant être accordée à la pensée de Lacan dans l'analyse de la série. Mais la question de l'altérité et de son lien avec le regard est présent chez les deux penseurs. Le regard lacanien suppose l'existence de ce que Lacan nomme le grand Autre, c'est-à-dire une entité externe omnisciente qui se porte garante de l'existence du sujet, du langage et de la culture. Parallèlement, le regard chez Foucault suppose lui aussi un regard omniscient qui assure une surveillance constante sur le sujet. Foucault illustre d'ailleurs l'omniprésence de cette surveillance par l'exemple du panopticon, une prison imaginée par le philosophe britannique Jeremy Bentham. Dans cette prison, les cellules sont disposées de façon circulaire, et s'ouvrent vers l'intérieur sur un espace dominé par une tour de garde; des volets sur la tour font en sorte qu'il est 
impossible pour le détenu de savoir quand il est observé par le garde, ce qui génère un sentiment de surveillance perpétuelle. Pour fonctionner, le pouvoir panoptique doit être à la fois visible et invérifiable : tout en apercevant en tout temps la tour d'où il peut être épié, le détenu ne doit jamais être en mesure de savoir si/quand il est épié. Dans cette mesure, comme Foucault le note bien, «le Panoptique est une machine à dissocier le couple voir-être $v u{ }^{13}$, une polarisation qui ne va pas sans rappeler la différence que Lacan note entre la vision et le regard. Pour Lacan, comme pour Foucault, le regard se pose comme la possibilité d'être vu par une entité externe qui nous échappe.

Dans l'un des rare articles visant à démontrer le pouvoir politique commun que partagent ces deux conceptions du regard, Henry Krips tisse des liens qu'il nous importe de souligner. Krips prend comme point de départ le sentiment anti-foucaldien qui anime les penseurs lacaniens contemporains dans le champ des études cinématographiques et médiatiques. Selon lui, le regard dans sa conception lacanienne partage plus de points communs avec celui de Foucault que ce qui est généralement reconnu. Pour tisser ce lien entre les deux concepts, l'auteur revient sur une anecdote relatée au sein du Séminaire XI, et qui mène à la conceptualisation lacanienne du regard. Le jeune Lacan est en excursion maritime avec un groupe de pêcheurs, quand l'un d'eux - le dénommé Petit-Jean - lui désigne un objet flottant au loin sur l'eau. L'objet, une boite de sardines, reflète le soleil. Petit-Jean dit au jeune Lacan, « Tu vois cette boite? Tu la vois? Eh bien, elle, elle ne te voit pas ", ce qui a pour effet de précipiter un sentiment d'inconfort et d'anxiété chez Lacan. Ce sentiment provient d'une part de l'incongruité de la situation dans laquelle Lacan se trouve : le jeune intellectuel privilégié est avec une famille de pêcheurs gagnant péniblement leur vie. Outre sa manifestation aveuglante, la réflexion de la boite de sardines crée un inconfort psychologique lié à la révélation d'un point de vue externe qui scrute. En repensant à la scopophilie freudienne, qui se décline sous une forme à la fois active et passive, on peut affirmer que la vision que Lacan dirige à l'extérieur rencontre une résistance dans la lumière éblouissante de la boite métallique ; la vision se retourne de façon réflexive vers Lacan, passant de l'actif au passif, transformant le «je vois » en « je suis vu ${ }^{14} »$. Comme le formule Lacan, « je ne suis pas simplement cet être punctiforme qui se repère au point géométral d'où est saisie la perspective. Sans doute, au fond de mon œil, se peint le tableau. Le tableau, certes, est dans mon œil. Mais moi, je suis dans le tableau $»^{15}$. Pour Lacan, le regard découle du positionnement du sujet dans un champ de vision qui échappe à son contrôle, et au sein duquel il peut être vu à partir d'une perspective qui s'élide.

8 Dans sa conception psychanalytique lacanienne, le regard représente donc une perspective externe posée sur le sujet. Comme le rappelle Krips, ce n'est pas tant un œil qui regarde le sujet, ni même un miroir dans lequel le sujet se voit en train de voir; au contraire, «c'est un point mort dans le champ visuel - dans le cas de la boite métallique, c'est un point où la perception se décompose et où ce qui constitue la perception, la lumière, devient visible ${ }^{16} »$. Comme le note McGowan, cette idée du regard comporte un antécédent philosophique chez Jean-Paul Sartre dans la mesure où le regard est le lieu d'une rencontre avec le grand Autre dans toute son absence ${ }^{17}$. "Le regard se voit précisément ce regard dont parle Sartre, ce regard qui me surprend, et me réduit à quelque honte [...]. Ce regard que je rencontre [...] est, non point un regard vu, mais un regard par moi imaginé au champ de l'Autre ${ }^{18}{ }^{\prime}$. C'est ici que Krips tisse des liens entre le regard lacanien et le regard foucaldien, notant que chez Lacan le point d'où se saisit la perspective externe du regard s'élide et nous échappe. Cette élision résulte de ce que le 
grand Autre n'existe pas, et que conséquemment il ne peut y avoir de point précis autour duquel gravite le regard - une problématique bien articulée dans Lost, comme nous allons le voir. Pour Krips, cette facette de la pensée lacanienne rejoint la pensée foucaldienne, puisque dans le contexte panoptique, tout le monde est sous surveillance, même le surveillant : « il n'y a pas de certitude absolue, il n'y a aucun point de vue divin à partir duquel une perspective fiable se révèle ${ }^{19} \%$. Une instance surveillante est donc imaginée au champ de l'Autre chez les deux penseurs. Dans une perspective globale, le regard reste le site d'une tension entre l'acte de voir (scopophilie active) et l'acte d'être vu (scopophilie passive). Bien que critiquable aux yeux des lacaniens ou des foucaldiens, le rapprochement tenté par Krips met en exergue certaines similarités qui peuvent s'avérer utiles dans l'examen de Lost, série qui semble dédiée à l'exploration de la thématique de l'identité et de l'altérité. Ces rappels sur le regard permettent de concevoir qu'il n'existe pas de position spectatorielle omnisciente possible; la vision soi-disant panoptique demeure un leurre dissimulant le point à partir duquel elle se retourne vers nous. Avec les précisions théoriques ici exposées, nous sommes à présent en mesure de nous pencher sur la série et, plus particulièrement, sur sa réception qui repose sur une radicalité pouvant se penser en termes à la fois philosophiques et psychanalytiques.

\section{Lost et la subjectivité à l'ère panoptique}

Dans Lost, ces deux conceptions du regard semblent opérer ensemble afin de générer l'expérience spectatorielle particulière de la série. En effet, les personnages sont constamment confrontés à une altérité aiguë qui prend plusieurs formes, et qui évolue au fil des dévoilements narratifs parsemant les six saisons. Parallèlement, dès leur rencontre avec les "Autres", ils sont soumis à diverses formes de pouvoir, qui ont comme dénominateur commun de reposer sur la surveillance et la dynamique panoptique. Nous pouvons aisément aborder la dynamique du regard et du pouvoir à travers trois facettes de la série : son articulation de l'altérité, son déploiement narratif du panoptisme, et sa problématisation de la position impossible du grand Autre.

\section{1) L'altérité}

10 Comme nous avons pu le constater précédemment, la notion de regard suppose l'Autre et ce, tant chez Lacan que chez Foucault. Il nous importe à présent de se centrer sur la déclinaison de l'altérité dans Lost telle qu'elle se manifeste par le biais de la médiation proposée par la série de cette notion de regard. L'altérité se révèle un moteur important dans la trame narrative de la série dès les premiers épisodes. En effet, le monstre de fumée - figure centrale de l'altérité dans la série - se manifeste dès le pilote, alors que les survivants s'organisent afin de passer leur première nuit sur la plage. De plus, au cours de la première saison, les survivants en viendront à découvrir qu'ils ne sont pas seuls sur l'île et feront plusieurs rencontres avec des formes variées d'altérité. Si le bruit et les cris du monstre de fumée éveillent un sentiment d'inquiétude chez les survivants dès le premier épisode, et si la mort du pilote dans le cockpit révèle également à certains personnages l'étrangeté de l'endroit où leur avion s'est écrasé, il reste que c'est plus tard au cours de la première saison que l'altérité prend une place définitive dans la thématique de la série. C'est notamment le cas dans l'épisode " Solitary " (S01E09), lorsque Sayid est confronté à la présence de Danielle Rousseau, qui le fait prisonnier. Cette première rencontre entre 
l'un des survivants et un personnage externe est cruciale, puisque c'est à partir de ce moment que les rescapés doivent concrètement faire face à la présence de l'autre présence préalablement signalée au spectateur par le biais de la médiation du regard, d'une perspective externe aux personnages. C'est d'ailleurs Rousseau qui, la première, parle de ces "Autres»; elle avoue ne les avoir jamais vus, mais les avoir entendus chuchoter dans la jungle. Au fil des épisodes, l'altérité des Autres est mise en avant, à la fois par le biais des chuchotements et de leur présence furtive, et finalement à travers leurs actions et la surveillance qu'ils exercent sur les survivants.

11 Les chuchotements jouent un rôle important dans le sentiment d'altérité qui se dégage de la série. Le fait qu'ils ne proviennent pas des Autres est moins important que le sentiment anxiogène qui en découle, et qui place le spectateur dans une tension manifeste avec la conception lacanienne du regard et du rapport à l'Autre. En effet, les chuchotements confrontent les personnages à la présence de l'Autre et, parallèlement, ils confrontent le spectateur à l'incomplétude du champ de vision qui lui est offert, et qui recèle nécessairement un point mort. Lorsqu'il se réfère à Sartre pour étayer l'idée d'un regard imaginé au champ de l'Autre, Lacan mentionne spécifiquement que la manifestation de ce regard transcende la dimension visuelle, ce qui permet de postuler qu'elle peut également être sonore. Comme il le formule, «Le regard dont il s'agit est bien [la] présence d'autrui comme tele ${ }^{20} »$; cette présence peut se manifester au-delà du champ visuel. S'appuyant sur cette précision, Krips précise d'ailleurs que, par ses manifestations sonores, «le regard correspond à un point de défaillance dans le champ visuel non pas parce qu'il éblouit, comme c'est le cas avec la boîte de sardines, mais parce que le sujet en devient conscient de façon sonore plutôt que visuelle ${ }^{21} »$. Il poursuit cette réflexion en ajoutant qu'une manifestation sonore du regard rappelle au sujet que même s'il n'y a rien à voir, il y a quelque chose de présent. "Ainsi, par le biais de moyens non-visuels, le sujet est amené à reconnaître qu'il y a une béance, une absence, dans son champ visuel - un quelque chose qui, en raison de sa présence imperceptible, fonctionne comme un point de défaillance du champ visuel ${ }^{22}$ ». Ainsi, que ce soit par les bruits du monstre ou par le biais des chuchotements qui se font entendre dans la jungle, le premier contact avec l'altérité dans Lost se fait sur le plan sonore, ce qui a pour effet de radicaliser le champ de l'Autre à la fois pour les spectateurs et pour les personnages. Malgré les dévoilements narratifs qui la marqueront, force est de constater la fascinante constance de cette thématique à travers l'évolution de la série. La position actantielle de l'altérité dans la série demeure constamment déclinée autour de la trame narrative, de sorte que le spectateur s'y trouve constamment confronté.

12 Au moment même où Sayid apprend l'existence des Autres sur l'île, Hurley termine son recensement et découvre qu'un des membres du groupe de survivants n'était pas à bord du vol 815. À partir de ce moment (à la moitié de la première saison), la thématique de l'altérité dynamise l'évolution de la trame narrative de façon irrévocable. L'altérité se fait d'abord sentir au sein même des survivants, par le biais de Sayid et de Locke: tous deux se présentent comme Autres par rapport au groupe. Alors que Sayid représente l'Autre comme celui qui provient d'une autre culture, Locke renferme une certaine qualité mystérieuse et énigmatique au fil de la première saison, notamment à travers son rapport avec Walt et Michael. La première rencontre avec les survivants de la queue de l'avion se passe également sous l'égide de l'altérité; une fois que les deux groupes de survivants seront réunis, la lutte contre les Autres se poursuivra, tout comme l'évolution de la figure de l'Autre. Finalement, le point charnière du rapport à l'Autre demeure la radicalité, qui 
découle de l'évanescence de la position même de l'Autre. L'altérité épouse effectivement plusieurs identités à travers les six saisons, et les terminologies utilisées renvoient à ce que la féministe Ann Kaplan qualifie de regard impérial. Pour Kaplan, les discours colonialistes sont nécessairement teintés d'un regard posé sur l'Autre à partir d'un système de valeurs qui lui est étranger. L'altérité est ainsi déterminée à partir de la position du sujet observant, et non de la personne observée. Comme elle l'explique, « les relations fondées sur la vision ne sont jamais innocentes. Elles sont toujours déterminées par les systèmes culturels apportés par les observants ${ }^{23}$ ». Si le groupe de Ben est désigné par les survivants comme les "Autres", Ben a pour sa part grandi au sein du projet Dharma, pour qui les habitants existants de l'île étaient qualifiés soit d'indigènes, soit d'hostiles. Les racines romaines de ces habitants de l'île sont elles-mêmes fondées sur l'altérité, la mère de Jacob demandant à ses fils d'entretenir une méfiance vis-à-vis des autres habitants de l'île. Dans tous ces cas, l'hostilité des relations est précédée d'une hostilité dans la réaction ressentie face à l'Autre dans toute son altérité et sa différence. Comme le précise Sarah Hatchuel, «La série met au jour la construction sociale et idéologique de l'altérité [...], nous fait partager la perspective des 'Autres' et révèle les rescapés comme des 'autres' des Autres : l'autre n'est jamais autre 'naturellement', mais parce qu'on l'a étiqueté et stigmatisé comme tel ${ }^{24} »$. Dans cette optique, les quelques répliques de Juliet, qui avoue qu'il est très "stressant d'être un Autre ", sont à prendre très au sérieux. Lost nous propose une perspective novatrice sur la dynamique du regard impérial, puisque la série permet à ce regard de se retourner vers l'observateur postcolonialiste.

13 Lost va même jusqu'à nous confronter à l'idée selon laquelle nous sommes tous l'Autre de quelqu'un; dans la série, dès qu'un Autre devient connu et qu'il cesse d'être menaçant, l'altérité se manifeste sous une forme renouvelée, et constamment transmise au spectateur par le biais d'une habile médiation du regard. À ce chapitre, il nous est même parfois donné de voir la perspective de l'Autre, ce qui nous permet de constater que les personnages auxquels nous nous identifions renferment également une forme d'altérité. Alors que les survivants se sont presque tous croisés dans leur vie antérieure, le renouvellement de l'altérité se perpétue jusqu'à ce que les survivants deviennent euxmêmes des Autres, des étrangers, dans la trame temporelle "parallèle » qui traverse la sixième saison. À ce stade de son déploiement narratif, la série met de l'avant la radicalité de l'altérité dans sa manifestation la plus triviale: dans cet autre que nous pourrions connaître, ou que nous aurions pu connaître dans un autre concours de circonstances.

\section{2) La surveillance panoptique}

14 Par ailleurs, si le regard occupe une place cruciale dans la détermination de l'altérité au sein de la série, ce même regard instaure également une importante dynamique panoptique qui structure le récit et notre rapport spectatoriel à celui-ci. L'étude de telles dynamiques panoptiques au sein des récits filmiques et télévisuels est au cœur de l'ouvrage Surveillance on Screen de Sébastien Lefait; cet ouvrage est fondé sur l'idée très juste selon laquelle la nature du lien entre surveillance et spectature mérite une attention plus poussée au sein des écrits savants ${ }^{25}$. L'auteur en vient à définir un certain « cinéma de la surveillance » (« surveillance cinema »), qu'il conçoit comme une forme filmique qui met en avant une réflexion sur le cinéma lui-même ${ }^{26}$. Même si ce n'est pas directement abordé dans l'ouvrage, il apparaît que cette dimension réflexive des récits de la surveillance repose entre autres sur la dynamique du regard et de son opposition à la 
vision qui est mis en scène. La perspective analytique offerte par Lefait peut conséquemment être agrémentée par les liens tissés ici entre les conceptions foucaldienne et lacanienne du regard. En effet, dans le cas qui nous occupe, Lost semble vouloir générer une forme de tension, chez ses spectateurs, entre la vision foncièrement voyeuriste propre au médium filmique et télévisuel, et l'apparition du regard dans toute sa radicalité. En commençant sur l'œil de Jack qui s'éveille suite au crash, et en montrant les yeux des personnages en ouverture d'un nombre conséquent d'épisodes, les créateurs de la série mettent d'emblée la vision en exergue. Cette vision fait écho à celle des spectateurs par le biais de l'identification aux personnages; dès lors, l'omniscience d'un regard posé sur les survivants à partir d'une perspective externe et furtive se fait sentir. Que ce soit par les plans en focalisation externe pris à partir d'une caméra au poing, ou par la présence sonore laissant transparaître l'existence d'une perspective externe insondable, la présence du regard que nous avons discutée jusqu'à présent repose sur la dynamique panoptique qui se déploie à travers la série. Rapidement, il devient évident que les survivants sont surveillés, mais nous ne savons pas par qui. Comme le dit Locke, « nous ne sommes pas les seuls sur cette île, et nous le savons tous » (S01E17) ${ }^{27}$. Certes, la présence en apparence omnisciente des Autres génère une forme de surveillance, mais ce même Locke est l'un des premiers à prendre connaissance d'un grand Autre qui surveille et qui structure leur existence sur l'île. Après sa rencontre avec le monstre dans l'épisode « Walkabout » (S01E04), il annonce à Jack dans l'épisode « White Rabbit » (S01E05) : «J'ai regardé dans l'œil de cette île, et ce que j'y ai vu était magnifique ${ }^{28} »$. Cette affirmation est loin d'être triviale: Locke affirme ainsi avoir aperçu l'instance à partir de laquelle l'île surveille ; il a été en contact avec la perspective impossible du regard qui se fait sentir dès la première saison, et qui persistera tout au long de la série. Il devient lui-même un Autre aux yeux de ses compatriotes en raison de cette perspective qui lui est offerte. Ainsi sommes-nous rapidement placés, dès le cinquième épisode de la première saison, devant l'idée d'une entité surveillante qui génère, à son tour, une altérité inquiétante menant à des rencontres avec le regard.

$15 \mathrm{Au}$ cours de la deuxième saison, les liens entre la série et le regard panoptique s'intensifient avec la découverte graduelle des stations Dharma. Dès les premiers plans de la deuxième saison, alors que la routine de Desmond est soudain interrompue par l'explosion de la trappe de sa station, c'est par le biais d'un dispositif complexe de miroirs que notre propre vision en vient à se poser sur Jack et Locke. Même le curseur du vieil ordinateur de la station semble évoquer un regard qui surveille. Au fur et à mesure que les survivants et les spectateurs découvrent les secrets des stations Dharma, les liens tissés entre Lost et le regard panoptique se déploient et se complexifient. La disposition même des stations Dharma sur l'île, ainsi que leurs interrelations complexes fondées sur la surveillance, semble renvoyer au panopticon de Bentham ${ }^{29}$. De plus, la surveillance ressentie durant la première saison se trouve plus profondément ancrée dans le récit à partir de la deuxième saison. Comme le note Amy Bauer, « La saison 2 de Lost semble aller au-delà du panopticon comme métaphore, adoptant l'expansion foucaldienne de la structure de Bentham comme dispositif structurel et dramatique ${ }^{30}$ ». L'importance de la surveillance au sein même de la structure narrative de la série prend donc une nouvelle importance avec l'approfondissement de nos connaissances sur l'île, les Autres et le groupe Dharma. D'ailleurs, le lien obscur des Autres avec le projet Dharma leur permettra de maintenir une altérité et une potentielle omniscience déconcertante aux yeux des 
survivants et des spectateurs; dans le même temps, la nature même de l'île comme structure panoptique est explorée et complexifiée.

La découverte de la station $n^{\circ} 5$, la Perle, participe de cette complexification de la dynamique panoptique. D'abord aperçue sur la carte dessinée par Stuart Radzinsky (S02E17, «Lockdown »), la Perle semble se situer au cœur de l'île, sous un immense point d'interrogation tracé à même le sol. Non seulement le point d'interrogation renvoie à la carte dessinée dans le Cygne, mais il est également mentionné dans le rêve de Mr. Eko au début de l'épisode «?» (S02E21), dans lequel la station Perle est finalement révélée. Dès leur arrivée dans la station, le logo Dharma apparaît. Cependant, alors que les logos des autres stations comportent en leur centre une forme donnée (une flamme, un cygne, etc.), le logo de la Perle - bien que tout à fait en adéquation avec le nom de la station - paraît vide. Ici, la ressemblance entre le logo Dharma et le panopticon devient cruciale, puisque le centre peut être considéré comme vide. Comme Sébastien Lefait le remarque, « ce qui rend Lost particulièrement pertinent dans le champ des études de la surveillance ne se résume pas à la présence diégétique de la surveillance dans la série, mais relève plutôt de la façon dont cette présence diégétique est convertie en un outil extradiégétique et réflexif ${ }^{31}$ ». Cette réflexivité est décuplée par l'approfondissement des connaissances sur la Perle qui marque les saison deux et trois de la série.

Comme Locke et Eko le découvrent, la Perle est une station de surveillance panoptique dans le sens le plus strict du terme. La vidéo d'orientation nous confirme même le rôle panoptique de la télévision et de notre propre position spectatorielle, puisque les deux partenaires travaillant dans la Perle se trouvent assis confortablement devant un mur de téléviseurs. Bien entendu, la position de surveillance est immédiatement mise à mal dans l'opposition qui s'ensuit entre Locke et Eko, et encore davantage au moment où nous comprenons que l'expérience n'était pas effectuée au frais des observés, mais des observateurs eux-mêmes. Au final, «avec l'intervention de la Perle comme preuve que certains personnages sur l'île sont sous observation sans le savoir, l'île semble être diégétiquement surveillée dans le cadre d'une expérience ${ }^{32}$ ", une affirmation qui ne va pas sans rappeler la posture foucaldienne voulant que «le panopticon est un lieu privilégié pour rendre possible l'expérimentation sur les hommes ${ }^{33}$ ». Située en plein centre de l'île, à l'instar de la tour de surveillance panoptique, la Perle s'avère le point central autour duquel les autres stations sont disposées de façon circulaire - du moins est-ce le cas dans la carte dessinée par Radzinsky. Désignée par un point d'interrogation renvoyant à l'identité nécessairement inconnue - et potentiellement inexistante - du surveillant, la Perle cristallise la problématisation de la position panoptique dans la série. La station nous confirme qu'il n'y a pas de position panoptique absolue, il n'y a que du leurre. Par ce fait même, la présence de cette station d'observation problématise également la position de spectateur face à la série, servant une fois de plus une médiation du regard mettant en avant une position spectatorielle fort novatrice.

Néanmoins, cette position panoptique trouble est encore plus profondément problématisée lorsque, dans la troisième saison, certains personnages retournent à la Perle pour tenter de communiquer avec d'autres stations. Se croyant dans la position de l'observateur, les personnages réussissent à voir des images de la station n ${ }^{\circ} 4$ (la Flamme) ; la caméra effectue un travelling panoramique pour révéler le visage inquiétant de Mikhail, qui retourne leur regard de son seul œil. La perspective panoptique traditionnelle s'en trouve profondément bouleversée, et la question de qui surveille et qui est surveillé refait surface de façon percutante. Pour emprunter les mots de Sarah 
Hatchuel, «dans Lost, des personnages sont surveillés, espionnés, manipulés par les images; d'autres surveillent, espionnent, manipulent, dans un brouillage permanent de qui observe qui et dans quel but ${ }^{34} »$. Dans cette cascade d'actes de surveillance, nous voyons également Ben devant un dispositif similaire, en train d'observer Jack, Kate et Sawyer. Pour un temps, nous sommes sous l'impression que Ben est le surveillant ultime, l'incarnation de l'autorité panoptique, celui qui contrôle les gestes et les événements. Mais si le regard panoptique procure une illusion de pouvoir à celui qui le détient, Ben demeure la première victime de cette illusion, lui qui tentera désespérément de maintenir un pouvoir qui lui échappe sans cesse. Il en va de même de la position spectatorielle déroutante proposée par la structure incessamment changeante de la série, qui nous prive d'une position de visionnement omnisciente. Si le panoptisme est étudié par Foucault pour asseoir sa notion de société disciplinaire, les nombreux artifices et tromperies utilisés par les Autres afin de contrôler les rescapés relèvent de cette notion de discipline - citons, par exemple, les déguisements et le faux campement qu'ils utilisent afin de laisser croire qu'ils ont un mode de vie primitif, ou la fumée noire qu'ils utilisent afin de prévenir de leur venue: ces dispositifs ont pour effet d'influer sur le comportement des rescapés. Le stimulateur cardiaque que Ben dit avoir installé dans le thorax de Sawyer afin de lui imposer calme et contrôle révèle un autre exemple de discipline s'appuyant sur une surveillance panoptique: comme le regard panoptique provient d'un Autre qui finalement n'existe pas, le stimulateur cardiaque n'a jamais été posé ; c'est l'effet du dispositif qui procure le pouvoir, et non le dispositif lui-même, qu'il soit cardiaque ou panoptique. Dans cette perspective, la série tient un discours complexe et complet sur la subjectivité, où la dimension panoptique du regard se marie à la radicalité de la position même de ce regard imaginé au champ de l'Autre.

\section{3) Jacob et la mort du grand Autre}

19 Si l'île s'avère une forme très complexe de panopticon, à la fois un «laboratoire du pouvoir » et un « lieu privilégié pour rendre possible l'expérimentation sur les hommes ${ }^{35}$ ", il faut admettre qu'il persiste toujours un angle mort au sein du panoptisme tel qu'il est abordé dans les analyses de Lost. C'est ici que l'intervention d'une perspective psychanalytique permet d'enrichir l'analyse foucaldienne mise en avant notamment par Sébastien Lefait. En effet, la position de la série comme fiction de surveillance permet de saisir ses nuances philosophiques, mais elle ne peut expliquer à elle seule la réception de la série. Comme nous avons pu le constater précédemment, la médiation du regard dans son sens lacanien intervient dans cette réception, et vient radicaliser le discours livré par la série sur l'altérité. Dans les relations entre les personnages de Lost, le passé de l'Autre vient déconstruire la notion même de l'altérité, puisque les personnages sont tous l'Autre d'un autre personnage ; au sein de la Dharma, le mystère plane sur ce qui se passe dans les autres stations ou entités : tout cela donne lieu à la présence sentie de ce que Lacan nomme le grand Autre. Cette présence est assurée par la médiation du regard offerte par la série, qui donne la possibilité d'un point à partir duquel se saisit une perspective qui élide à la fois le spectateur et les personnages de la série. Éventuellement, ce grand Autre sera doté d'un nom : Jacob. Et, bien que Jacob ne soit mentionné pour la première fois que dans la troisième saison, la thématique du grand Autre traverse la série dans son ensemble. Car si l'idée d'un être supérieur arrive à mi-chemin dans Lost, sa présence se fait sentir dès le début de la série. D'emblée, tous les rescapés traînent un lourd passé qui agit comme une tache dans leur existence - certains plus que d'autres, si l'on pense à 
Kate, ou même à Jin ou à Ben. Ils se trouvent coincés entre l'idéal de ce qu'ils devraient/ voudraient être et ce qu'ils sont en réalité. Un tel contraste confronte nécessairement le sujet à l'origine des idéaux qu'il tente d'atteindre, qui ne proviennent pas de lui mais de ce que Lacan nomme l'ordre symbolique, c'est à dire la dimension culturelle de notre existence, qui repose sur la signification, la Loi et le grand Autre.

Cette logique lacanienne se manifeste de façon limpide dès la première saison, à travers la déclaration de Jack concernant la nécessité de vivre ensemble sans quoi les rescapés mourront seuls. Dans la pensée lacanienne, le grand Autre garantit l'existence du sujet. Autrement formulé, si le sujet existe, c'est parce que le grand Autre veut quelque chose de lui, une situation qui s'avère intenable et conséquemment génératrice du désir. Lacan illustre la position du sujet dans cette impasse par la question italienne "che vuoi? », qui signifie " que me veux-tu? ", une question dont l'absence de réponse génère le désir. Le sujet structure son désir autour de cette énigme, convaincu que le grand Autre attend quelque chose de lui, qu'il existe pour une raison ou pour accomplir quelque chose. Dans Lost, les rescapés pourraient aisément diriger cette question vers Jacob, qui visiblement veut quelque chose d'eux - Jack le fait d'ailleurs de façon frappante dans l'épisode «Lighthouse » (S06E05), lorsqu'il comprend qu'il a effectivement été observé toute sa vie par Jacob, et que ce dernier l'a délibérément mené sur l'île pour une raison qu'il ne peut saisir. Véritable tour panoptique, le phare place Jack dans la position impossible de l'Autre qui observe, rendant cette séquence particulièrement cruciale pour la série dans son ensemble, et plus particulièrement pour la dynamique du regard qui y est déployée depuis le tout début, et qui se dévoile ici dans une séquence paroxystique. Devant le constat selon lequel il a été observé toute sa vie par Jacob, et voulant savoir pourquoi il a été ainsi surveillé, Jack pose la question à Hurley: «Que veut-il de moi ${ }^{36}$ ?» Face à l'absence de réponse à cette question fondamentale, Jack procède à la destruction des miroirs au travers desquels le phare lui procure la perspective impossible du grand Autre. Jack veut des explications, mais il n'en obtiendra pas - pas plus que Ben qui, avant de tuer Jacob, tente une dernière fois d'obtenir la justification de son existence en lui demandant «Et moi dans tout ça?" La réponse de Jacob, «Quoi, vous?", renvoie directement à l'aspect aléatoire de l'existence, le «che vuoi?» lacanien ne trouvant aucune réponse satisfaisante ${ }^{37}$.

21 Mais le trait central qui structure le grand Autre dans la pensée de Lacan demeure son inexistence, d'où l'idée de vivre ensemble puisque la mort nous frappe inévitablement dans la solitude la plus totale ${ }^{38}$. La logique du fantasme vient pallier le vide généré par l'absence de réponse à «che vuoi? » en proposant des pistes de réponse. Le fantasme se pose alors comme la solution de l'énigme: le sujet existe parce que ses parents l'ont désiré, par exemple, ou bien car il est destiné à accomplir de grandes choses. Le rôle du fantasme ici est de faire miroiter au sujet une réponse à cette question intenable; il fournit ainsi au sujet une piste de sortie devant l'impasse liée à la pure contingence de sa propre existence. Dans cette optique, la logique du fantasme est au centre du conflit entre Jack et Locke: Jack prône une approche scientifique et empirique face aux diverses manifestations étranges liées à la vie sur l'île, refusant la logique du fantasme, alors que Locke se laisse guider par cette logique, convaincu qu'il y a un grand Autre qui lui veut quelque chose. Si Jack est convaincu de la nécessité de la vie en groupe puisque la mort frappe inévitablement le sujet dans la solitude, Locke ne craint pas cette solitude, convaincu qu'il est d'exister pour une raison. La force de Lost est de maintenir une réponse ambiguë à l'impasse du «che vuoi? ». Jacob agit bel et bien comme une forme de 
grand Autre, garantissant aux rescapés que leur existence est fondée sur un désir, qu'ils sont là pour une raison; mais finalement, Jacob est un humain qui a commis ses propres erreurs, et qui subit lui-même la tare du passé et de ses origines. Il n'est pas en mesure de prévenir sa propre mort, et subit les contrecoups de ses propres erreurs et de son existence incomplète. Il dévoile ainsi la défaillance de la position impossible du grand Autre.

C'est à travers cette problématisation de la position du grand Autre que la série se révèle à nous sous son jour le plus radical au plan psychanalytique. La série semble nous indiquer qu'il est vain de chercher le grand Autre au-delà de soi, et qu'il serait plutôt pertinent d'accepter la contingence de notre propre existence. Cette suggestion est notamment transmise par le déploiement du regard dans toute sa radicalité, un déploiement qui se manifeste de façon particulièrement frappante lorsque notre relation avec le regard est reconfigurée. Cette reconfiguration, qui se manifeste à de nombreuses reprises par un repositionnement des perspectives, a lieu notamment au moment où Sawyer est le témoin de la naissance d'Aaron (S05E04). Scène glanée au fil des flashs qui le propulsent à travers le temps, cette vision est bouleversante pour lui ainsi que pour les spectateurs. Certes, elle l'est en raison de ce qu'il ressent pour Kate, mais sa radicalité est ailleurs: elle découle de l'aspect non naturel d'une telle perspective sur un moment qui ne lui était pas destiné. Il adopte, le temps d'un instant, la position impossible du « regard imaginé au champ de l'Autre ». Autrement dit, Sawyer adopte la position de Jacob, alors que l'omniscience et la toute-puissance de Jacob s'avèrent être un leurre. Cette dynamique se reflète bien entendu dans la réception de la série, qui nous confronte aux aspects les plus radicaux de nos propres relations avec l'altérité. Dans cette mesure, on peut affirmer que Lost porte bien son nom : nous sommes tous des sujets «perdus » dans les dédales de nos relations. La médiation du regard, comme il a été possible de le constater ici, permet donc de remettre en question notre propre perspective sur les relations et sur l'altérité en tant que telle. Dans cette mesure, Lost aura sans doute ouvert la voie à de futures réflexions médiatiques sur notre rapport au regard et à son origine.

\section{BIBLIOGRAPHIE}

BAUER Amy, « Discipline \& Punish: The Lost Experience as Panopticon », Lost Online Studies 1.2, 2006, http://loststudies.com/1.2/discipline.html.

BUCKLAND Warren (éd.), Puzzle Films: Complex Storytelling in Contemporary Cinema, Malden (MA), Wiley-Blackwell, 2009.

COWIE Elizabeth, Representing the Woman: Cinema and Psychoanalysis, Minneapolis, University of Minnesota Press, 1997.

DENBY Richard, « The New Disorder: Adventures in Film Narrative », The New Yorker, 5 mars 2007.

FOUCAULT Michel, Surveiller et punir. Naissance de la prison, Paris, Gallimard, 1975.

FREUD Sigmund, Trois essais sur la théorie sexuelle, Paris, Gallimard, [1905] 1987. 
HATCHUEL Sarah, Lost : Fiction vitale. Paris, Presses universitaires de France, 2013.

KAPLAN Ann E., Looking for the Other: Feminism, Film, and the Imperial Gaze, New York, Routledge, 1997.

KLECKER Cornelia, « Mind-Tricking Narratives: Between Classical and Art-Cinema Narration », Poetics Today 34.2, 2013, p. 119-146.

KRIPS Henry, « The Politics of the Gaze: Foucault, Lacan, Žižek », Culture Unbound 2, 2010, p. 91-102.

LACAN Jacques, Séminaire XI. Les quatre concepts fondamentaux de la psychanalyse, Paris, Seuil, 1973.

LEFAIT Sébastien, Surveillance On Screen : Monitoring Contemporary Films and Television Programs, Toronto (ON), The Scarecrow Press, 2013.

LOMBARDI Giancarlo, « Everything You Always Wanted to Know About Lost but Were Afraid to Ask Lacan, Derrida, and Foucault », in Looking for Lost: Critical Essays on the Enigmatic Series, éd. Randy Laist, Jefferson, McFarland, 2011, p. 90-104.

MCGOWAN Todd, « Looking for the Gaze: Lacanian Film Theory and Its Vicissitudes », Cinema Journal 42.3, 2003, p. 27-47.

. The Real Gaze: Film Theory After Lacan, New York, SUNY Press, 2007.

METZ Christian, Le signifiant imaginaire, Paris, Christian Bourgois, [1977] 2002.

MULVEY Laura, « Visual Pleasure and Narrative Cinema », Screen, 16.3, 1975, p. 6-18.

WILLIS Louis-Paul, « Vers un 'Nouveau Hollywood' ? Considérations sur la métalepse dans le cinéma populaire contemporain », Kinephanos 2.1, 2011, p. 67-88.

\section{NOTES}

1. Nous entendons par le terme "narrations complexes » un corpus de films contemporains qui rejette les conventions plus linéaires propres au cinéma hollywoodien afin de plonger le spectateur dans des récits à la fois non-linéaires, fragmentés et comportant plusieurs trames narratives. De plus en plus populaires depuis les années 1990, les narrations complexes font l'objet d'études depuis une dizaine d'années. Voir notamment Warren, Buckland (éd.), Puzzle Films: Complex Storytelling in Contemporary Cinema, Malden (MA), Wiley-Blackwell, 2009 ; Richard Denby, "The New Disorder: Adventures in Film Narrative ", The New Yorker, 5 mars 2007 ; Cornelia Klecker, " Mind-Tricking Narratives: Between Classical and Art-Cinema Narration ", Poetics Today 34.2, 2013, p. 119-146 ; et Louis-Paul Willis, "Vers un 'Nouveau Hollywood' ? Considérations sur la métalepse dans le cinéma populaire contemporain », Kinephanos 2.1, 2011, p. 67-88.

2. Les ouvrages abordant la similarité entre le panoptisme et l'univers de Lost sont nombreux; nous aborderons dans ce qui suit certaines de ces réflexions, plus spécifiquement celles de Amy Bauer, "Discipline \& Punish: The Lost Experience as Panopticon », Lost Online Studies 1.2, 2006, http://loststudies.com/1.2/discipline.html ; Giancarlo Lombardi, «Everything You Always Wanted to Know About Lost but Were Afraid to Ask Lacan, Derrida, and Foucault ", in Looking for Lost: Critical Essays on the Enigmatic Series, éd. Randy Laist, Jefferson, McFarland, 2011, p. 90-104 ; et Sébastien Lefait, Surveillance On Screen: Monitoring Contemporary Films and Television Programs, Toronto (ON), The Scarecrow Press, 2013.

3. Certes, ces notions échappent presqu'assurément aux spectateurs, mais elles déterminent néanmoins la radicalité de leur expérience devant une série télévisuelle comme Lost. Il importe 
donc de les démystifier afin de mieux comprendre comment la série peut être explorée à travers elles.

4. Plaisir lié à la vision et à la monstration.

5. Dans ses Trois essais sur la théorie sexuelle (Paris, Gallimard, 1987 [1905]), Freud rattache effectivement la pulsion scopique au « plaisir de regarder-et-de montrer » (p. 119) et précise les deux pôles de la scopophilie : le voyeurisme et l'exhibitionnisme.

6. Laura Mulvey, « Visual Pleasure and Narrative Cinema », Screen, 16.3, 1975, p. 9.

7. La notion d'une position spectatorielle «toute-percevante » provient de Christian Metz qui, dans Le Signifiant imaginaire, propose une réflexion psychanalytique du cinéma.

8. Jacques Lacan, Séminaire XI. Les quatre concepts fondamentaux de la psychanalyse, Paris, Seuil, 1973, p. 98.

9. Texte original: « The gaze is not the look, for to look is merely to see whereas the gaze is to be posed by oneself in a field of vision", Elizabeth Cowie, Representing the Woman: Cinema and Psychoanalysis, Minneapolis, University of Minnesota Press, 1997, p. 288.

10. Texte original : «the pleasure of the scopic drive is first and foremost passive - the wish to be seen (in one's sex, finally) ", ibid.

11. Texte original : " desire here is not the wish to see some particular thing, or even just to see, but to see what is being shown, what I cannot yet see but know is there to see ", ibid.

12. Texte original : «the gaze is not the look of the subject at the object, but the point at which the object looks back », Todd McGowan, «Looking for the Gaze: Lacanian Film Theory and Its Vicissitudes ", Cinema Journal 42.3, 2003, p. 28-29.

13. Michel Foucault, Surveiller et punir. Naissance de la prison, Paris, Gallimard, 1975, p. 235.

14. Henry Krips, «The Politics of the Gaze: Foucault, Lacan, Žižek », Culture Unbound 2, 2010, p. 93.

15. Lacan, p. 110-111.

16. Texte original : « it is a point of failure in the visual field - in the case of the tin can, a point where perception breaks down and the stuff out of which perceptions are constituted, namely light, becomes visible », Krips, p. 93.

17. Pour une analyse finement détaillée du regard et de son rôle dans l'analyse du cinéma, voir Todd McGowan, The Real Gaze: Film Theory After Lacan, New York, SUNY Press, 2007.

18. Lacan, p. 98.

19. Texte original : "there is no absolute certainty, no God's eye point of view from which a trustworthy picture is revealed », Krips, p. 97.

20. Lacan, p. 98.

21. Texte original : " here the gaze corresponds to a point of failure in the field of the visible not because (as in the case of the tin can) it dazzles the eye, but rather because the subject becomes aware of it aurally rather than visually ", Krips, p. 94.

22. Texte original : "Thus, by totally non-visual means the subject is brought to recognize that there is a hole, a lack, in his visual field - a something that, because it is present but cannot be seen, functions as a point of failure of the visual field ", ibid.

23. Texte original : "looking relations are never innocent. They are always determined by the cultural systems people travelling bring with them », Ann E. Kaplan, Looking for the Other: Feminism, Film, and the Imperial Gaze, New York, Routledge, 1997, p. 6.

24. Sarah Hatchuel, Lost: Fiction vitale. Paris, Presses universitaires de France, 2013, p. 106.

25. Sébastien Lefait, Surveillance on Screen: Monitoring Contemporary Film and Television Programs, Toronto, Scrarecrow Press, 2013, p. vii.

26. Lefait, p. xiv.

27. Texte original : «We're not the only ones on this island and we all know it. »

28. Texte original : « I've looked into the eye of this island, and what I saw... was beautiful. »

29. Voir Lombardi, p. 90-104. 
30. Texte original: "Season 2 of Lost appears to move beyond the Panopticon as metaphor, adopting Foucault's expansion of Bentham's structure as both a structural and dramatic device ", Amy Bauer, "Discipline \& Punish: The Lost Experience as Panopticon », Lost Online Studies 1.2, 2006, http://loststudies.com/1.2/discipline.html.

31. Texte original : « What makes Lost particularly relevant in the field of surveillance studies [...] is not the way it includes surveillance as a diegetic element, but the way this diegetic element is turned into an extradiegetic, reflexive tool », Lefait, p. 118-119.

32. Texte original : « with the intervention of the Pearl station as proof that some characters on the island are under watch without knowing they are, the island appears as diegetically surveilled for the purpose of an experiment ", Lefait, p. 121.

33. Foucault, p. 238.

34. Hatchuel, p. 29.

35. Foucault, p. 238.

36. Texte original : "What does he want from me ?». Cette question rappelle de façon frappante le «che vuoi ?» lacanien.

37. Dans le dialogue original, Ben demande à Jacob « What about me? ", ce à quoi Jacob répondra «What about you? », perpétuant l'énigme du «che vuoi? » de façon d'autant plus frappante.

38. Dans la version originale, Jack répète souvent l'adage « live together, die alone ».

\section{RÉSUMÉS}

Parmi les nombreuses références à la philosophie qui se déclinent au sein de son univers narratif, Lost est notamment traversé par une thématique prédominante pouvant être rattachée à la pensée de Michel Foucault, la dynamique du regard et de la surveillance marquant l'un des aspects les plus notoires de la série. Les thèmes propres au panoptisme et aux sociétés disciplinaires fondent effectivement plusieurs pans cruciaux de l'intrigue, et plusieurs analyses de la série en font aisément la démonstration. Cet article se propose pour sa part d'examiner les répercussions spectatorielles liées à cette thématique, qui traverse les six saisons de Lost, en tissant des liens entre le regard panoptique et le regard dans sa conception psychanalytique, élaborée par Jacques Lacan. En analysant ces deux regards déployés au champ de l'Autre, il est ensuite possible d'aborder Lost comme un discours télévisuel qui explore la nature même de l'existence subjective à l'ère du panoptisme. En explorant l'importance et le déploiement de ces concepts dans la série, il s'avère possible de constater la radicalité qu'elle articule autour de la notion de l'altérité, ainsi que son expression par le biais de la médiation du regard.

Among the numerous references to philosophy that permeate its narrative universe, Lost seems to be predominantly traversed by Foucauldian themes, mostly related to articulations of looking dynamics and surveillance. Themes related to panopticism and discipline constitute notable underpinnings for the series' plot, as several previous analyses have pertinently demonstrated. This article proposes an examination of the spectatorial repercussions related to this facet of Lost , most importantly by establishing ties between the panoptic gaze and the gaze in its psychoanalytic conception, elaborated by Jacques Lacan. By examining both these concepts of a gaze deployed around the position of the Other, this article focuses on the series' exploration of subjectivity in the era of panopticism. By exploring the importance and narrative deployment of these notions of the gaze within Lost, it is suggested that the series articulates a certain radicality 
around the notion of Otherness, as well as its expression through the televisual mediation of the gaze.

\section{INDEX}

Mots-clés : Lost, regard, altérité, Lacan Jacques, Foucault Michel, psychanalyse, médias Keywords : Lost, gaze, otherness, Lacan Jacques, Foucault Michel, psychoanalysis, media

\section{AUTEUR}

\section{LOUIS-PAUL WILLIS}

Louis-Paul Willis est professeur d'études cinématographiques et médiatiques à l'Université du Québec en Abitibi-Témiscamingue. Ses intérêts de recherche portent sur la psychanalyse, le féminisme, le genre, les théories de la réception, et les questions du regard, du désir et du fantasme au cinéma. En plus de nombreux articles et chapitres d'ouvrages collectifs, il a récemment codirigé l'ouvrage collectif Žižek and Media Studies: A Reader, ainsi qu'un numéro sur la traversée des fantasmes écraniques pour la revue en ligne Écranosphère et un numéro sur Lacan et la théorie du cinéma pour la revue CiNéMAS.

Louis-Paul Willis is professor of film and media studies at the Université du Québec en AbitibiTémiscamingue. His research interests focus on psychoanalysis, feminism, gender, reception theories and questions pertaining to the gaze, desire and fantasy in cinema. He has published several essays in various journals and collected editions; he has also recently co-edited Žižek and Media Studies: A Reader, and has acted as guest editor for an issue on the traversal of screen fantasies for Écranosphère, as well as for an issue on Lacan and film theory for CiNéMAS. 